\title{
Amplified Fiber-Optic Recirculating Delay Lines
}

\author{
Beatriz Vizoso, Carmen Vázquez, Rafael Civera, Manuel \\ López-Amo, Member, IEEE, and Miguel A. Muriel, Member, IEEE
}

\begin{abstract}
Experimental and theoretical results on single- and double-amplified recirculating delay lines are presented. One of our aims is to emphasize their application as filters, showing a wide flexibility of design. Analysis of their performance in the spectral and time domains have been carried out. A novel method of understanding the behavior of double structures has been developed and successfully tested with experimental results employing Er-doped fiber amplifiers as delay lines.
\end{abstract}

\section{INTRODUCTION}

$\mathbf{F}$ IBER-OPTIC signal processing devices can be constructed to perform various functions, such as convolution, correlation [1], pulse-train generation, matched-filtering [2], data-rate transformation and limitation [3] or frequency filtering [4], [5].

The signal-processing properties of all-fiber devices have also been utilized for the development of sensors. One of the most interesting configurations of fiber sensors is the recirculating delay line [6]. This configuration uses the recirculating character of the optical circuit to create phase-bias regions of high measured sensitivity [7].

A disadvantage of these kinds of sensors is the need for very low loss in the optical circuit to obtain a sharp resonance [8]. In order to compensate the loss in these sensors or signal processors, rare-earth-doped fiber amplifiers can be inserted in the delay lines.

The effect of the amplifiers is the addition of new control parameters that enable the tailoring of the frequency response of the structures. Depending on the main parameters of the amplifiers (gain and saturation output power), the resulting circuit may exhibit certain advantages in comparison with the classical passive-delay loops, as suggested in [5] and [9].

In this paper we discuss the performance of single and double all-fiber recirculating delay lines when optical amplifiers are introduced. Theoretical and experimental results are shown utilizing commercially available light sources and detectors. The effective coherence length of the light source utilized was much shorter than the length of the loops we have mounted. Consequently, the interference that produces the frequency dependence is the interference of the modulation, not the interference of the optical carrier, as in [10] or [11]. The light power at a given modulation frequency is modified by the amplifying delay line.

Manscript received May 13, 1992; revised November 9, 1992. This work was supported by Spanish CICYT (TIC-211/89), CAM-PRI (C059/90) and UPM (Acciones concertadas).

The authors are with the Departamento 'de Tecnología Fotónica. E.T.S.I Telecomunicación, Ciudad Universitaria, 28040-Madrid, Spain.

IEEE Log Number 9209881

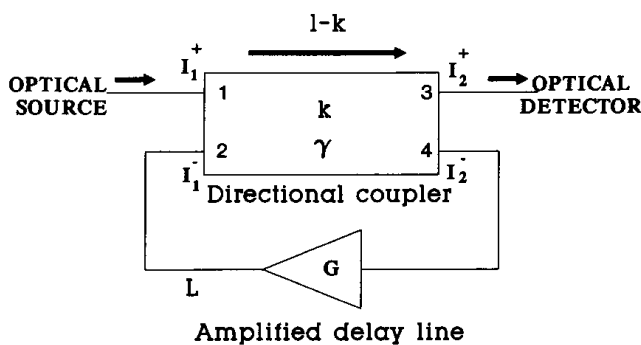

Fig. 1. Simple recirculating fiber-optic processor, introducing an Erbiumdoped fiber acting as delay-line. $I_{j}^{s}$ are the optical field intensities at the input/output ports of the directional coupler, numbered from 1 to 4 .

It is our aim that further understanding of the behavior of these kinds of structures may create new applications.

\section{Single-Mode FibER RECIRCULATING Delay LINES INCLUDING FIBER AMPLIFICATION}

A schematic diagram of a fiber recirculating delay line is shown in Fig. 1. The structure is supposed to be excited by a light source with a broad spectrum, providing that the frequency components of the optical carrier will beat against each other and be time-averaged in the detector at the output of the structure [5]. The fiber that connects output $2^{-}$and input $1^{-}$of the directional coupler is supposed to be a rare-earthdoped fiber, with an electrically controlled optical gain $G$ and a total length $l$, which includes the directional coupler pigtails.

Conventional semiconductor laser diodes can exhibit both a broad output spectrum and the ability to be directly modulated at speeds up to several gigahertz-the sources of choice for most optical fiber delay-line applications.

\section{A. Calculation of the Transfer Function of the Structure}

When an optical carrier as mentioned above is modulated by an RF signal, the system constituted by the source, the modulator, and the optical fiber structure are involved as in an electronic processor. The system is lineal with the intensity, because the output of the fiber structure is proportional to the sum of the powers obtained from the amplitude-modulated light source and the delayed signal that recirulate from the output $2^{-}$to the other input $1^{-}$of the directional coupler.

Because of the linearity of the intensity, the calculation of the transfer function may be carried out with the intensity equations of the directional coupler as follows:

$$
\begin{aligned}
& I_{2}^{+}=(1-\gamma)(1-k) I_{1}^{+}+(1-\gamma) k I_{1}^{-} \\
& I_{2}^{-}=(1-\gamma) k I_{1}^{+}+(1-\gamma)(1-k) I_{1}^{-}
\end{aligned}
$$


The recirculating line of the structure gives the condition:

$$
I_{1}^{-}=G I_{2}^{-} F e^{-j \Omega \tau}
$$

where:

- $\gamma$ is the fractional loss of the directional coupler

- $k$ is the coupling ratio of the directional coupler

- $G$ is the optical gain introduced by the rare-earth-doped fiber amplifier/delay line.

- $I_{1}^{+}, I_{1}^{-}, I_{2}^{+}, I_{2}^{-}$are the optical intensities at the input/output ports of the coupler, as shown in Fig. 1.

- $F=10^{-\alpha l_{o} / 10}$

- $\alpha$ is the propagation loss per unit length, expressed in $d B / m$

- $\tau$ is the propagation delay line.

- $l_{o}$ is the length of the unamplified fiber $(m)$.

- $\Omega$ is the electrical modulation frequency (RF signal).

The delay loop is composed by two different types of fibers, one that amplifies the optical signal (rare-earth-doped fiber) and the other one, the unamplified fiber, that in most cases will be the pigtails of the directional coupler. In commercially available amplifiers, the length of the doped fiber is between 15 and $40 \mathrm{~m}\left(l_{1}\right)$ and the pigtails of the directional coupler are about $1 \mathrm{~m}$ long on each port. Taking into account these lengths, (3) becomes:

$$
I_{1}^{-}=G I_{2}^{-} 10^{-\alpha\left(l_{o}\right) / 10} e^{-j \Omega\left(\tau l+\tau_{o}\right)}
$$

where:

- $\tau_{1}=\left(n_{1} l_{1}\right) / c$ is the temporal delay introduced by the doped-fiber.

- $n_{1}$ is the refraction index of the doped-fiber.

- $c$ is the light speed in the vacuum.

- $\tau_{o}=\left(n_{o} l_{o}\right) / c$ is the temporal delay introduced by the unamplified fiber.

- $n_{o}$ is the refractive index of the unamplified fiber.

Therefore, typical difference between doped-fiber refractive index and coupler fiber refractive index is no greater than two hundredths and $l_{1}$ is greater than $l_{o}$. Thus, in the following calculations we are going to consider $n_{1}=n_{0}=n$ and $l=l_{1}+l_{0}$, consequently utilizing (3) instead of (4) with $F=10^{-\alpha\left(l_{0}\right) / 10}$ and $\tau=n l / c$.

Operating with the expressions (1)-(3), the following transfer function is obtained:

$$
T=\frac{I_{2}^{+}}{I_{1}^{+}}=(1-\gamma)\left[\frac{(1-k)+M(2 k-1)}{1-M(1-k)}\right]
$$

where:

$$
M=G^{*} Z^{-1}
$$

being $G^{*}=F G(1-\gamma)$ and $Z=e^{j \Omega \tau}$

The structure of Fig. 1 has been studied in several papers [12], [13].

The transfer function is periodic in frequency. The frequencies where the maxima are located are given by:

$$
f=\frac{m c}{n l}
$$

where $m=0,1,2,3, \ldots$ and $f$ is the frequency.
The frequencies of the minima are:

$$
f=\frac{(2 m+1) c}{2 n l}
$$

In order to provide a physical interpretation of (7), we may consider the structure of Fig. 1 and assume a sinusoidal RF input signal of frequency $f_{1}$. This results in an output signal that is the sum of the input signal multiplied by a factor and the different contributions of the feedback ring. All of these signals will add, giving maximum output amplitude when they add in phase.

The frequency distance between maxima is:

$$
\Delta f=c / n l
$$

The utilization of this structure as a filter is possible until certain frequency $\left(f_{x}\right)$. For higher frequencies the relative difference between maxima and minima is too small. This frequency, $f_{x}$, depends on the parameters of the system.

\section{B. Dependence of the Transfer Function on} the Parameters of the Structure

The characteristics of this system are defined by four main parameters. Changes in any of them introduce variations in the transfer function that may be utilized to reshape it in one way or another, depending on the different applications.

- Changes in the length of the ring

These changes introduce two different variations:

1. Ring loss decreases or increases because of the variation of the factor $F=10^{-\alpha l_{0} / 10}$.

2. The frequencies of the maxima and minima of the transfer function change. The modification of the optical length of the delay loop without changing the "physical" length of the fibers is feasible by means of a phase modulation that can be induced by a phase modulator in the delay loop or operating on the refractive index of the delay fiber. Therefore, the change of this parameter implies an additional complication of the structure of Fig 1.

- Changes in the gain of the delay-loop.

The analysis of the changes introduced by the variation of the optical gain of the delay-loop fiber has to be completed by the study of the "stability" of the system.

The pole $(p)$ and zero $\left(z_{c}\right)$ of the transfer function (5) are determined by the expressions:

$$
\begin{aligned}
z_{c} & =\frac{F G(1-\gamma)(2 k-1)}{(k-1)} \\
p & =F G(1-\gamma)(1-k)
\end{aligned}
$$

We have centered the measurements in three particular cases. The experimental setup is as shown in Fig. 1. The input light to the system is a CW LD at $1.5 \mu \mathrm{m}$ sinusoidally modulated in frequency with a RF tracking generator. The loop is constructed with a $2 \times 2 k$ variable polarization preserving fiber coupler, and its pigtails of port $2^{-}$and port $1^{-}$are connected with an Erbium-doped fiber amplifier pumped at $1480 \mathrm{~nm}$. The total length of the delay loop was $40 \mathrm{~m}$. 
The variable coupler was perfectly characterized by means of measuring the coupling coefficient and the fractional loss for each position of the micropositioner. Taking into account that $k=P_{4} /\left(P_{3}+P_{4}\right)$ and $(1-\gamma)=P_{3} /\left[(1-k) P_{1}\right]$ where $P_{3}$ and $P_{4}$ are the output intensities at port 3 and 4 , respectively, and $P_{1}$ is the input intensity at port 1 (see the port number assignation in Fig. 1). The values of $P_{1}, P_{3}$ and $P_{4}$ have been set on a table for each micropositoner value and have been used to determine every $k$ and $\gamma$. This last one oscillates around $0.05 \pm 0.005$.

The last factor to characterize this configuration is the overall gain of the loop $\left(G^{*}\right)$, which is calculated from the value of $k$. The method for calculating $\left(G^{*}\right)$ from $k$ consists of forcing any of the next three possible spectral behaviors of the structure, which allows us to obtain the relation between the two parameters directly:

1) $z_{c} \rightarrow-1$

$$
k=\frac{G^{*}+1}{2 G^{*}+1} \text { so } G^{*}=\frac{(1-k)}{(2 k-1)}
$$

This case is of particular interest due to its flat response and sharp decaying at certain frequencies, as shown in Fig. 2(a) and (b). Both characteristics reinforce its application as a notch filter. It is important to point out the influence of the losses $\left(G^{*}\right)$ in the critical value of $k$ to achieve the optimum spectral response, apart from the fact that can be usually compensated with the adequate value of $G$.

2) $p \rightarrow+1, z_{c} \rightarrow-1$

$$
\begin{aligned}
k & =2-\sqrt{2} \\
G^{*} & =\sqrt{2}+1
\end{aligned}
$$

The condition $p \rightarrow+1$ imposes the proximity of the system to the unstable area. This was reflected in the problem of obtaining a stable figure at the laboratory. High peaks of the transfer function can be observed in Fig. 3 with $p=0.9931, k=0.586$ and $G^{*}=2.399$. An interesting application of this filter would be as a notch filter for the zero frequencies and a selective one for the frequencies around the maxima.

3) $p \rightarrow+1, z_{c}$ far away from -1

$$
k=\frac{G^{*}-1}{G^{*}} \text { so } G^{*}=\frac{1}{(1-k)}
$$

We can consider a situation in which the main objective is to achive high maxima without any interest in the suppression of a certain set of frequencies. Specific applications can be as a frequency demultiplexing. Calculations and experimental results are shown in Fig. 4.

After analyzing the gain influence, it can be concluded from experimental results that these filtering structures present problems to give a stable figure close to the unit circle. Such a situation may be caused by light source instability, environmentally induced loop-length fluctuations. But the main problems are those related to the amplifier itself: Amplified Spontaneous Emission (ASE) and its resonance (when the loop behave as a laser resonator cavity: $\left.G_{0}=k(1-\gamma) F G=1\right)$.

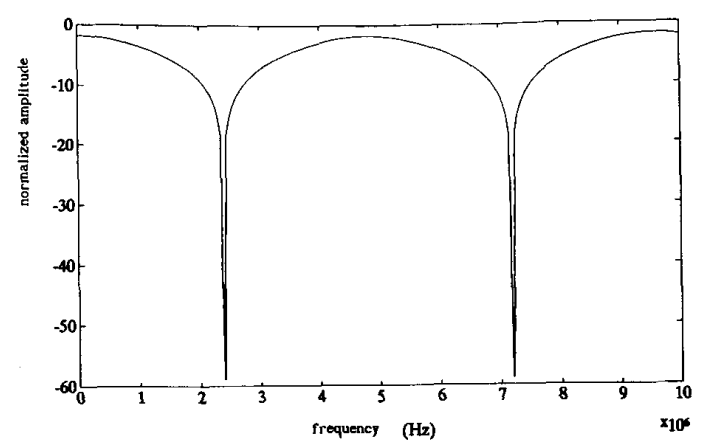

(a)

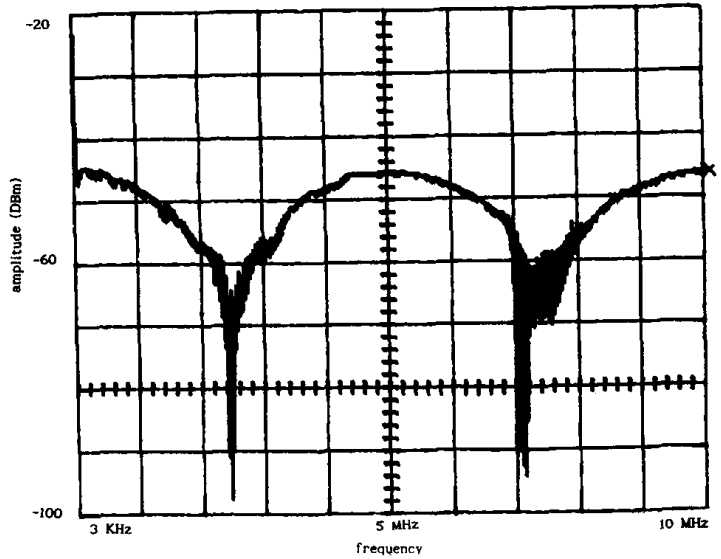

(b)

Fig. 2. Case 1: Single amplified recirculating delay line $z_{c}=-1$, $p=0.1659, k=0.7261, G^{*}=0.6058$. (a) Calculations. (b) Experimental results.

Both can be partially avoided by using a filter of several $\mathrm{nm}$ around the signal wavelength [14]. Stimulated Brioullin backscattering (SBS) is overcome by the presence of an optical isolator into the optical amplifier. And, finally, amplifier gain has to be stabilized. The lack of this last condition derives in fluctuations around the bounds of the unit circle with the consequent oscillatory behavior of the system. The dynamic range of the system is limited by two factors: input signal has to be high enough to avoid ASE, but not so high that the amplifier enters its saturation region (as it would be analyzed in the stability section).

- Changes in the coupling ratio

From (11), the stability condition of the system is given by:

$$
G^{*}(1-k) \leq 1
$$

If the remaining parameters of the structure are fixed, there is a value of the coupling ratio $k\left(k_{s}\right)$, under which the system becomes unstable. $k_{s}$ gives also the maximum amplitude peak value of the transfer function. Increasing $k$ beyond $k_{s}$, the maximum decreases.

As was seen, the absolute minimum is reached when the system parameters satisfy the zero condition (10). Changes in $k$ introduce the following variations in the minima: 


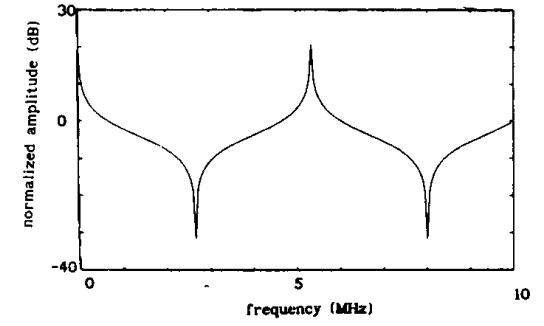

(a)

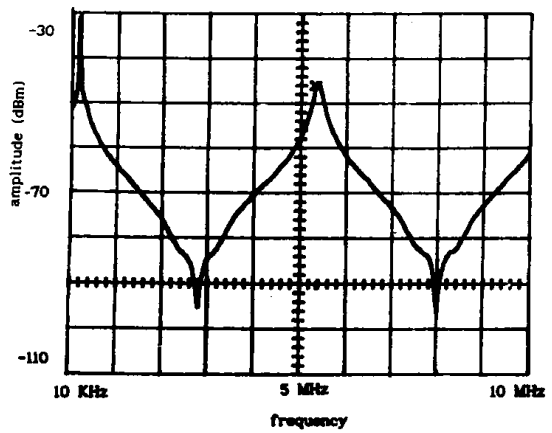

(b)

Fig. 3. Case 2: Single amplified recirculating delay line. $z_{c}=-1, p=0.9931, k=0.586, G^{*}=2.399$.(a) Calculations. (b) Experimental results.

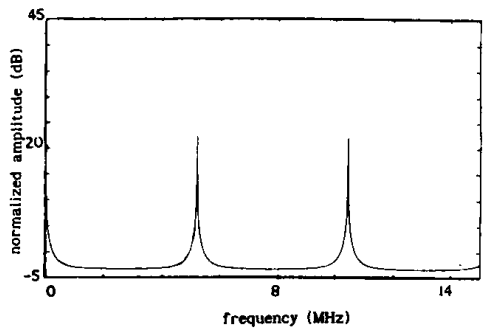

(a)

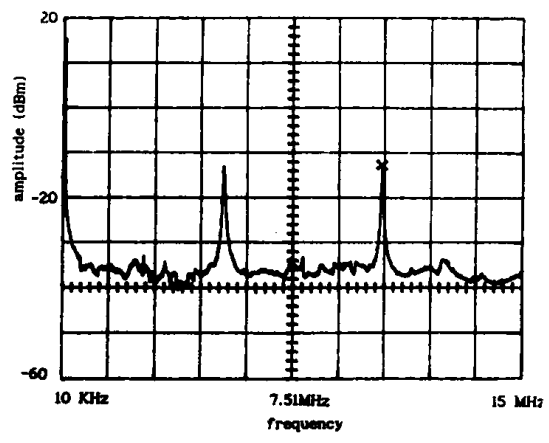

(b)

Fig. 4. Case 3: Single amplified recirculating delay line configuration. $z_{c}=-0.5546, p=0.9984, k=0.46, G=1.664$. (a) Calculations. (b) Experimental results.

* If $\frac{(2 k-1)}{(1-k)} G^{*}<1$, by increasing $k$ and maintaining the rest of the parameters unaltered, the value of the minimum decreases.
* If $\frac{(2 k-1)}{(k-1)} G^{*}>1$, by increasing $k$ and maintaining the rest of the parameters unaltered, the value of the minimum increases.

\section{Stability of the System}

In Section II(B), we described some stability characteristics of the system. Now, we will emphasize that the identification of the pole of the transfer function (11) provides the theoretical stability system condition. Fig. 5(a) shows the case with $p<1$, so the response of the system to a narrow pulse input is a decaying amplitude pulse sequence, while in Fig. 5(b) $p=1$, so the pulse sequence presents a uniform amplitude and the system can be considered as a pulse generator. These measurements have been taken with an oscilloscope when the system was excited with a CW LD at $1.55 \mu \mathrm{m}$ modulated in frequency with a narrow pulse. To implement the narrow pulse input physically, a pulse train has been used. Each pulse has width $\tau_{1}=40 \mathrm{~ns}$, which is very small-in comparison with the loop delay time $\tau=200 \mathrm{~ns}$ and a very large period $T=20 \mu s$ - to analyze for a long enough time. So if the system is analyzed in frequency, the maxima are higher when it reaches the condition $p=1$, but, from the point of view of time analysis, the proximity to $p=1$ results in a considerable increment of the transient time (previous to the steady state) of the system. Finally, it can be concluded that the increment of the output amplitude of the system leads to a reduction in its speed response. A compromise between both (maxima amplitude and speed response) must be achieved for each specific application.

\section{System Response to Different Input Signals}

The system responds to any signal through a transient time prior to its steady state. This last state can only be reached by those systems that follow the stability condition $p \leq 1$. We will analyze this process for different input signals.

- System Response to a Periodical Nonsinusoidal Signal

As it was shown in Section II(A), the transfer function given by (5) is periodic, its repetition rate determined by $\tau$. If the input signal has a period of $T_{0}$, depending on the ratio $\frac{\tau}{T_{0}}$, the system response shows different behaviors. A number of particular cases of interest have been analyzed:

$$
i-\quad \tau=n T_{0} \text { where } n=1,2,3, \ldots
$$

The output signal follows the input signal once the steady state of the system has been reached, but its amplitude is multiplied by the maximum amplitude of the transfer function. This structure is well suited for amplifying periodical signals. If (15) is satisfied, output signal does not suffer distortion because the harmonic frequencies of the periodical signal are located where the transfer function presents their maxima. It is also interesting to notice how the amplitude changes every $n$ periods of the signal (Fig. 6) during the transient state.

$$
i i-T_{0}=n \tau \text { where } n=2,3,4 \ldots
$$

The system distorts the input signal. This distortion depends on $n$ (Fig. 7(a)). If $n \geq N_{p}$, where $N_{p}$ is the maximum 


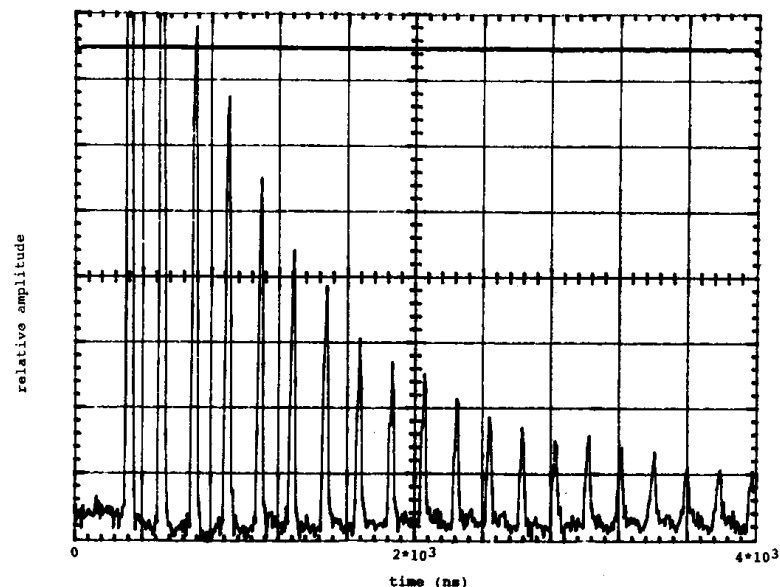

(a)

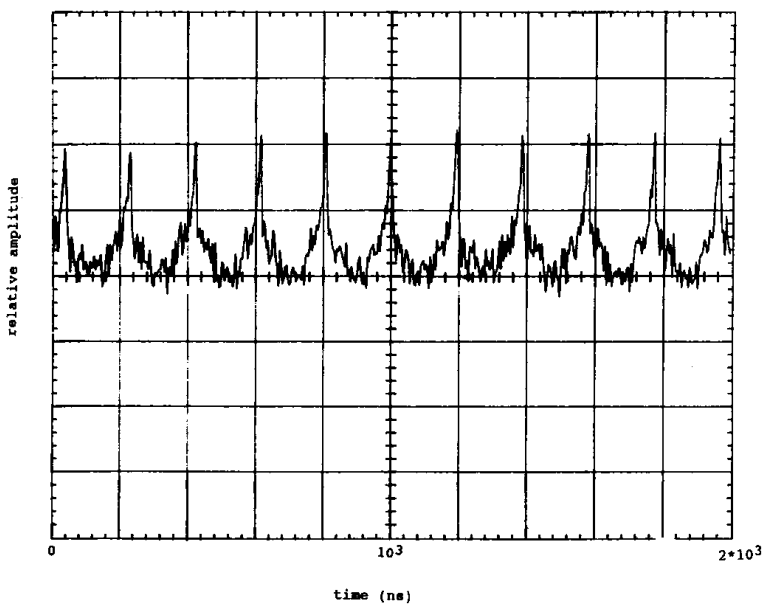

(b)

Fig. 5. System response to a sequence of pulses with period $T$ and pulse width $\tau\left(T=20 \mu \mathrm{s}, t=40 \mathrm{~ns}<t_{\text {loop }}\right.$ [loop delay time]= 200ns ) with $k=0.15$ for (a) $p<1$, (b) $p=1$.

number of times that the signal can be feeedbacked before we get a negligible amplitude, the system follows the input signal without distortion. In other words, the frequency of the input signal has to be low enough to allow all the significant harmonics to be within the first period of $H(\Omega)$ (Fig. 7(b)).

$$
i i i-\tau=Q T_{\mathrm{o}}
$$

where $Q$ and $\frac{1}{Q}$ are non-integer numbers.

In this case, the harmonic frequencies of the periodical signal are not going to fit the peaks of the transfer function, consequently its spectral components would be multiplied by different amplitudes of the transfer function. Therefore, if (17) is satisfied, the system distorts the input signal shape, as shown in Fig. 8.

- System response to a nonperiodical signal

In this section we will analyze the output signal of this kind of structures if a nonperiodical input signal with a maximum spectral frequency $f_{\max }$ is introduced.

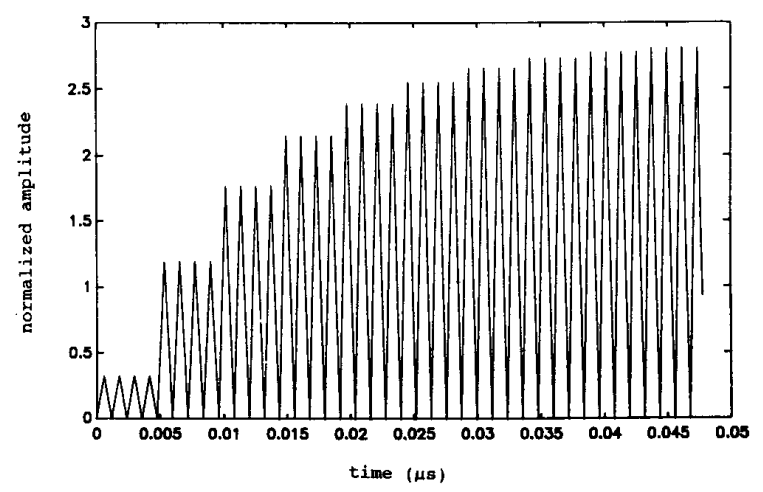

Fig. 6. System response to a normalized triangular periodic input of period $T$ related to the delay time $\tau$ of the loop by the relation $\tau=4 T, G=2, k=2 / 3, l=1 m, \gamma=0.05, \alpha=0.0005 \mathrm{~dB} / \mathrm{m}$, $n=1.44$, showing its transient response until the steady state is reached.

If a nondistorted output signal is desired, the available bandwidth of the transfer function is reduced to the band located around the origin and it imposes a limit to $f_{\max }$ given by the ring length $\left(\tau N_{p}=\frac{1}{f_{\max }}\right)$. If $l=20 \mathrm{~m}$ the maximum frequency $\left(f_{\max }\right)$ must be less or equal than $f_{\max }=20 \mathrm{MHz}$.

Other kind of nonperiodical signals are the data sequences. Due to their importance, we will treat them separately in next section.

\section{- System response to a sequence of pulses}

The analytic expression of a sequence of $n$ terms separated by an interval $T$ with the information ( 1 or 0 ) located in $C_{m}$ can be represented as follows:

$$
e(t)=\sum_{m=0}^{n} C_{m} p(t-m T), \text { being } C_{m}=1 \text { or } 0
$$

where $p(t)$ is the signal that bears the transmission of the bit information. In this analysis $p(t)$ is a square pulse.

The behavior of this structure is based on the interference of the different recirculations. So to achieve the value of $H(\Omega)$ for a certain frequency at the time response, it should be considered a finite number of terms at the summatory of $h(t)$, which can be labeled $N_{p}$. This $N_{p}$ will increase when the system is close to the instability (higher values of $H(\Omega)$ ); this will happen with the interference between two consecutive bits. This compromise between $N_{p}$ and $G^{*}$ can be expressed through the relation:

$$
\left[G^{*}(1-k)\right]^{N_{p+1}}<<1
$$

To treat this kind of signals, an amplitude modulation will be considered: a sinusoidal carrier and a square pulse modulator signal. Both are related by the condition:

$$
f_{\text {carrier }}(\text { minimum })=N_{p} f_{\text {modulator }}
$$

The value of $f_{\text {carrier }}$ depends upon the selection made in (19), which must be based on the desired characteristics of the structure. On the other hand, there is a decrease in the maximum frequency of the modulator signal proportional to the increase of $N_{p}$, which means that a compromise between the maximum of $f_{\text {modulator }}$ selected and the gain of $H(\Omega)$ 


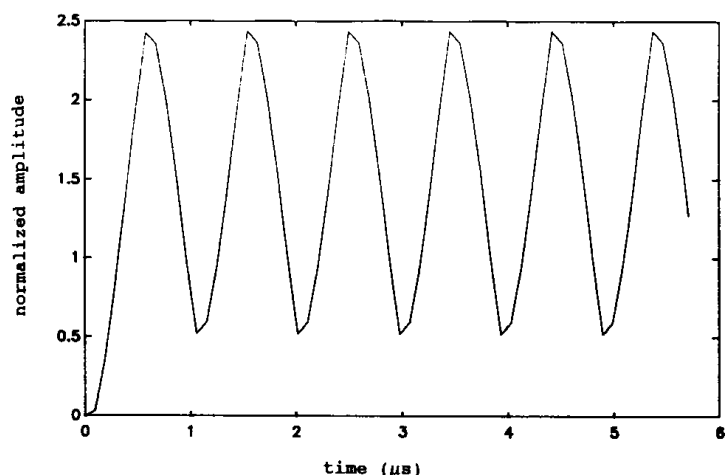

(a)

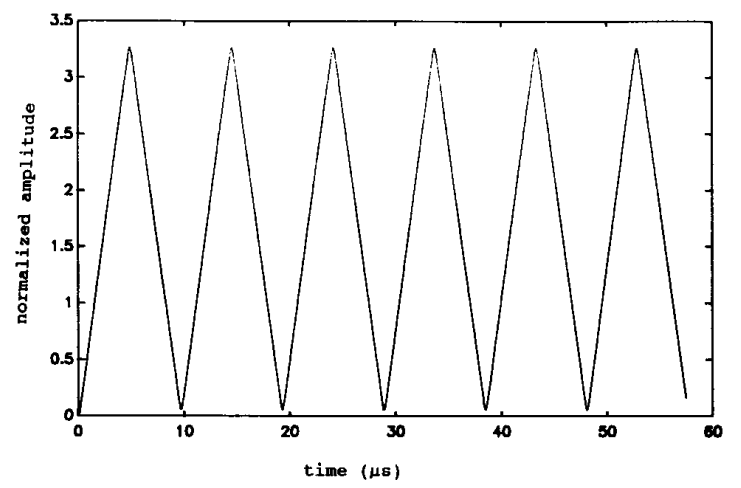

(b)

Fig. 7. Output intensity against time for a normalized triangular-shape input signal of period $T$ related to the delay loop by the relation: (a) $T=10 \tau$ (distortion appears). (b) $T=100 \tau$ (no distortion). In both cases, $G=2.5, k=0.8, \alpha=0.0005 \mathrm{~dB} / \mathrm{m}, \gamma=0.01, n=1.44$.

has to be reached. Another important factor is that the output signal can not be sampled at any time along the bit period. It has to be done after a guard time, which is also dependent on $N_{p}$. Finally, for low values of $N_{p}$ a margin definition of 1 and 0 should be needed. Two calculations are shown in Fig. 9 for the same value of $G^{*}$ and higher amplification (a), with $f_{\text {modulator }}=20.833 \mathrm{MHz}$ :

$$
G^{*}(1-k)^{N_{p+1}}=4.883 \times 10^{-4}<<1
$$

and Fig. 9(b), for $f_{\text {modulator }}=41.66 \mathrm{MHz}$ :

$$
G^{*}(1-k)^{N_{p+1}}=2.44 \times 10^{-4}<<1
$$

Finally the output sequence is shown in Fig. 9(c) when its carrier frequency is located in a minimum of the transfer function of the system. During the transient times of the bit change, the signal is not attenuated, but this would be avoided if the guard times were used when sampling the output sequence.

- System response including the saturation output power of the amplifier

The optical amplifier can be saturated by a specific power and it has to be taking into account when the system response to different inputs is analyzed. The best way to do

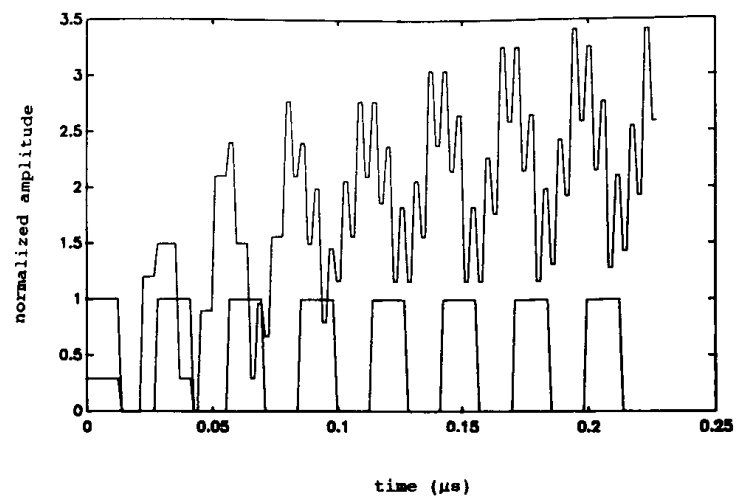

Fig. 8. Output intensity against time for a normalized square input signal of period $\tau$ related to the loop delay time $t$ by the expression $T=1.19 \tau$. The remainder parameters are: $G=2.5, k=0.7, l=5 \mathrm{~m}, \gamma=0.01, \alpha=0.0005$ $\mathrm{dB} / \mathrm{m}, n=1.44$.

so is by showing different experimental results in which this characteristic is observed. The gain curve of optical amplifiers against signal input is almost flat for a certain range of input power signal (to define a saturation output power, $-3 \mathrm{~dB}$ gain compression), and for input power signals beyond that limit, the gain decays progressively. In other words, the gain of the amplifier depends upon the value of the input signal to the amplifier. For filtering purposes we pretend in this work, a constant gain is required if saturation is not wanted, which means the input power signal must be limited to a certain range that depends on the coupler coefficient and losses.

Fig. 10(a) shows the case of an unsaturated behavior, and in Fig. 10(b) the saturation region is reached and the pulses with enough amplitude are cut in amplitude. As in the analysis of the stability of the system, this has been excited with a very narrow pulse (pulse train with $T \gg \tau$ ).

\section{Double Recirculating RING STRUCTURE}

The structure we will analyze in this section is depicted in Fig. 11. This structure is excited by an incoherent optical source. As in Fig. 1, the optical amplification can be achieved by means of the delay-loop lines, if they are assumed to be Erbium- $\left(E r^{3+}\right)$ doped fiber pumped with a laser diode.

\section{A. Calculation of the Transfer Function of the Structure}

In order to calculate the transfer function of a double recirculating ring structure, the equations of the directional couplers and the conditions imposed by the recirculating rings are given by:

$$
\begin{aligned}
& I_{2}^{+}=\left(1-\gamma_{1}\right)\left(1-k_{1}\right) I_{1}^{+}+\left(1-\gamma_{1}\right) k_{1} I_{1}^{-} \\
& I_{2}^{-}=\left(1-\gamma_{1}\right) k_{1} I_{1}^{+}+\left(1-\gamma_{1}\right)\left(1-k_{1}\right) I_{1}^{-} \\
& I_{4}^{+}=\left(1-\gamma_{2}\right)\left(1-k_{2}\right) I_{3}^{+}+\left(1-\gamma_{2}\right) k_{2} I_{3}^{-} \\
& I_{4}^{-}=\left(1-\gamma_{2}\right) k_{2} I_{3}^{+}+\left(1-\gamma_{2}\right)\left(1-k_{2}\right) I_{3}^{-} \\
& I_{3}^{-}=G_{3} 10^{-\left(\alpha 3 l_{p} / 10\right)} e^{-j \Omega \tau 3} I_{4}^{-} \\
& I_{3}^{+}=G_{1} 10^{-\left(\alpha 1 l_{p} / 10\right)} e^{-j \Omega \tau 1} I_{2}^{-} \\
& I_{1}^{-}=G_{2} 10^{-\left(\alpha 2 l_{p} / 10\right)} e^{-j \Omega \tau 2} I_{4}^{+}
\end{aligned}
$$




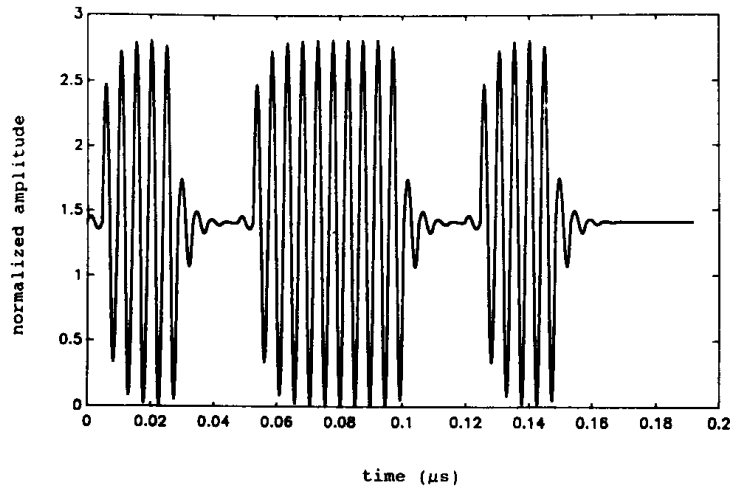

(a)

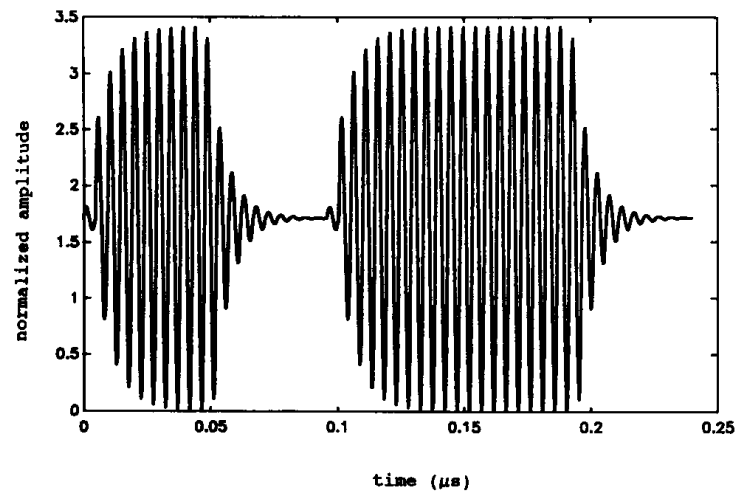

(b)

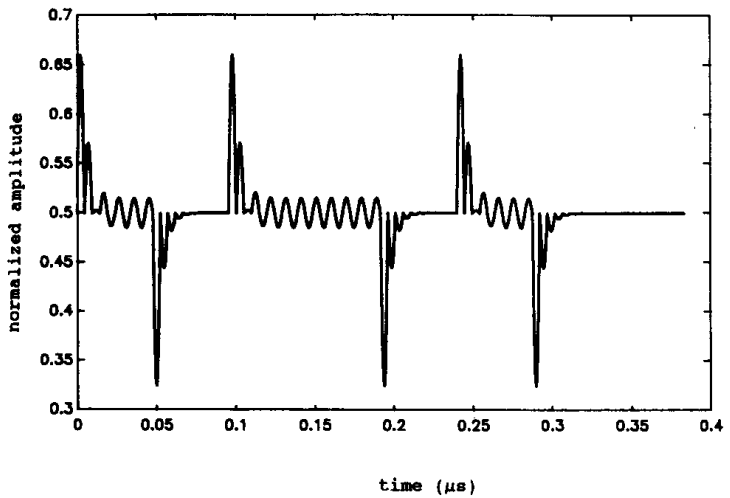

(c)

Fig. 9. Output intensity against time for a normalized pulse amplitude modulated sequence with: (a) $f_{\text {carrier }}=208.32 \mathrm{MHz}, f_{\text {modulator }}=41.66 \mathrm{MHz}$ (which means $N_{p}=5$, so $\left[G^{*}(1-k)\right]^{N p+1}=2.4410^{-4}<<1$ ) and the parameters of the structure are: $k=0.9, G=2.5, l=1 m, \alpha=\gamma=0$. (b) $f_{\text {carrier }}=208.32 \mathrm{MHz}, f_{\text {modulator }}=20.833 \mathrm{MHz}$ (that is $N_{p}=10$, so $\left[G^{*}(1-k)\right]^{N p+1}=4.883 \times 10^{-4}<<1$ while with $N_{p}=5$, $\left[G^{*}(1-k)\right]^{N p+1}=0.0156<1$ but not $\left.<<1\right)$ and the parameters of the structure are: $k=0.8, G=2.5,1=1 m, \alpha=\gamma=0 .(\mathrm{c}) f_{\text {carrier }}=104.162$ $\mathrm{MHz}, f_{\text {modulator }}=28.83 \mathrm{MHz}$, and the parameters of the structure are $G=1, k=0.68,1=1 m, \alpha=\gamma=0$

where:

* $\gamma_{1}, k_{1}, \alpha_{1}$ are the coupler fractional losses, the coupling ratio, and the fiber attenuation of the first directional coupler

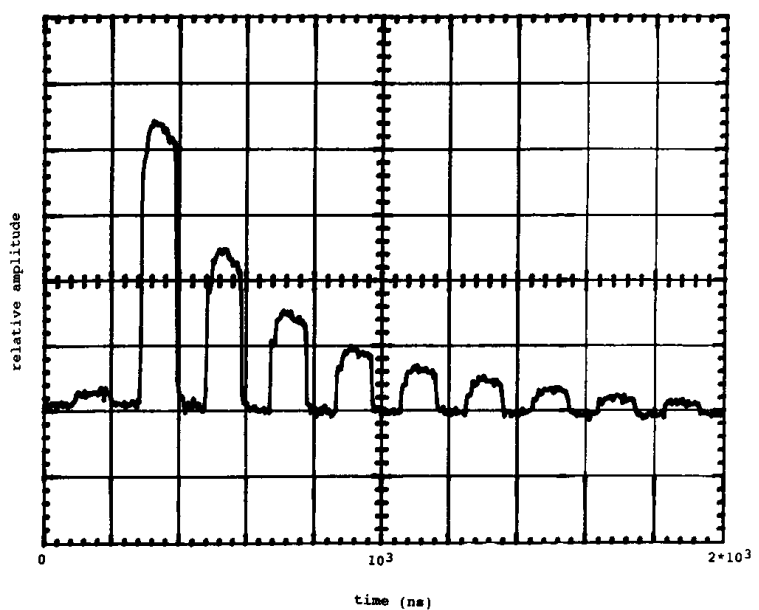

(a)

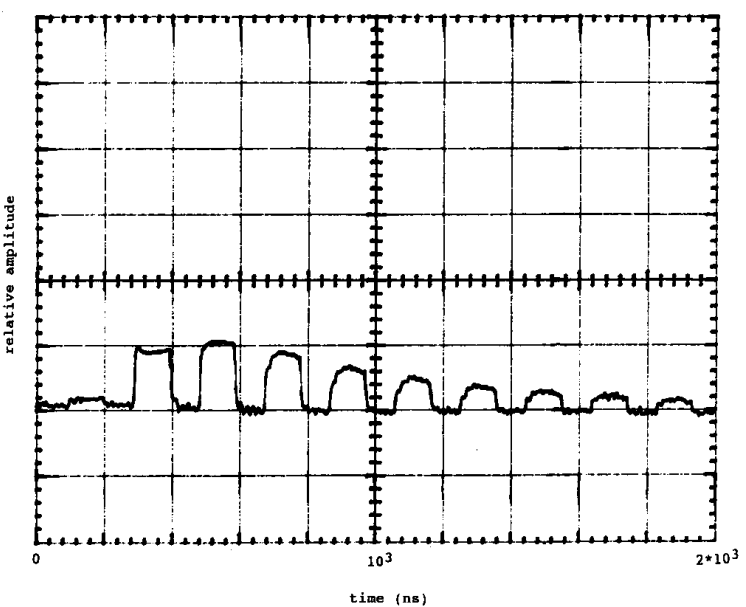

(b)

Fig. 10. System response to a pulse train of $T_{1}=20 \mu \mathrm{s}$ and pulse width $\tau_{i}=$ $40_{\text {ns }}<$ loop delay time $\tau=200_{\mathrm{ns}}, k=0.11, \alpha=0.00003 \mathrm{dBm}, \gamma=0.05$, $n=1.44$, changing $G$ to work within : (a) nonsaturation region of the amplifier, (b) saturation region of the amplifier.

indicated if Fig. 11, respectively, and $\gamma_{2}, k_{2}, \alpha_{2}$ are the corresponding parameters of the second directional coupler, also indicated in Fig. 11. $l_{p}$ is the sum of any two pigtails when all of them are of identical length. In the event that there would be any other unamplified fiber to be considered, an $l_{p i}$ will take it into account where the subindex "i" is related to each loop.

* $G_{1}, G_{2}, G_{3}$ are the gain introduced in each one of the fiber loop lines. $\tau_{1}, \tau_{2}, \tau_{3}$ are the propagation delay times introduced by these loop lines (Fig. 11).

$* \Omega$ is the electronic frequency ( $\mathrm{RF}$ signal).

$* I_{1}^{+}, I_{1}^{-}, I_{2}^{+}, I_{2}^{-}, I_{3}^{+}, I_{3}^{-}, I_{4}^{+}, I_{4}^{-}$are the intensities noted in Fig. 11.

The transfer function obtained from (23)-(29) is given by:

$$
\begin{aligned}
H(\Omega)= & \left(1-\gamma_{1}\right)\left(1-k_{1}\right) \\
& +\left(1-\gamma_{1}\right) k_{1} \frac{A S_{1} S_{2}\left(1-\gamma_{1}\right) k_{1}}{1-\left(1-\gamma_{1}\right)\left(1-k_{1}\right) A S_{1} S_{2}}
\end{aligned}
$$




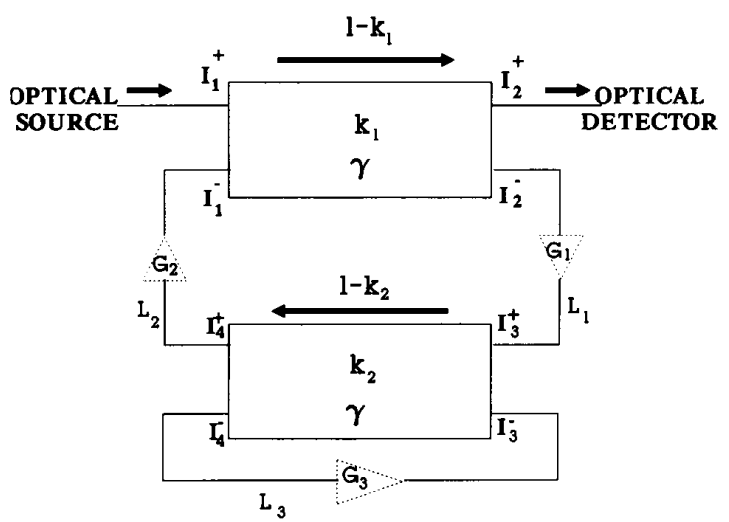

Fig. 11. Double recirculating fiber optic processor, introducing an Erbium-doped fiber acting as delay-line in any of the lines of this structure. $I_{j}^{s}$ are the optical field intensities at the input/output ports of the directional couplers.

where:

$$
\begin{gathered}
S_{i}=G_{i} 10^{-\left(\alpha i_{p} / 10\right)} e^{-j \Omega \tau i} ; i=1,2,3 \\
A=\left(1-\gamma_{2}\right) \frac{\left(1-k_{2}\right)+\left(1-\gamma_{2}\right)\left(2 k_{2}-1\right) S_{3}}{1-\left(1-\gamma_{2}\right)\left(1-k_{2}\right) S_{3}}
\end{gathered}
$$

The transfer function (30) is periodic in frequency and presents a series of maxima and minima. There is one frequency in each repetition period at the location of the absolute maximum.

Regarding the secondary maxima, it can be mentioned that the number of them in a repetition period is governed by the ratio of the lengths of the rings.

\section{B. Analysis of the Transfer Function}

While in the single ring the well known $z$ transformation was used to determine the location of zeros and poles of the transfer function, such an application cannot be used to analyze (30). So a novel method is now proposed in this paper to locate the maxima/minima of this configuration. This new method consists of splitting up the system into three single, independent rings:

1. The first ring is constituted with $l_{1}$ and $l_{2}$ as it is shown in Fig 12(a).

2. The second ring corresponds to $l_{3}$ (Fig. 12(b)). The whole transfer function is multiplied by a factor of $k_{1}^{2} G_{1} G_{2}$ due to the first and last cross through coupler 1 in each turn.

3. The last one includes $l_{1}, l_{2}$ and $l_{3}$ (Fig. 12(c))

Each of these rings has maxima at the frequencies that are located in:

(1) $\tau_{1}+\tau_{2}=N_{1} T_{1} \rightarrow f_{1}=N_{1} /\left(\tau_{1}+\tau_{2}\right)$

(2) $\tau_{3}=N_{2} T_{2} \rightarrow f_{2}=N_{2} / \tau_{3}$

(3) $\tau_{1}+\tau_{2}+\tau_{3}=N_{3} T_{3} \rightarrow f_{3}=N_{3} /\left(\tau_{1}+\tau_{2}+\tau_{3}\right)$,

$$
\text { where } N_{1}, N_{2}, N_{3} \in \mathbb{N}
$$

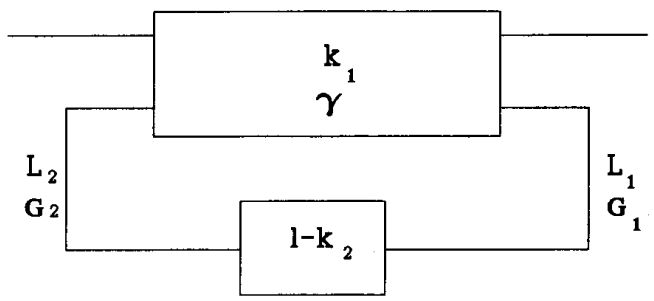

(a)

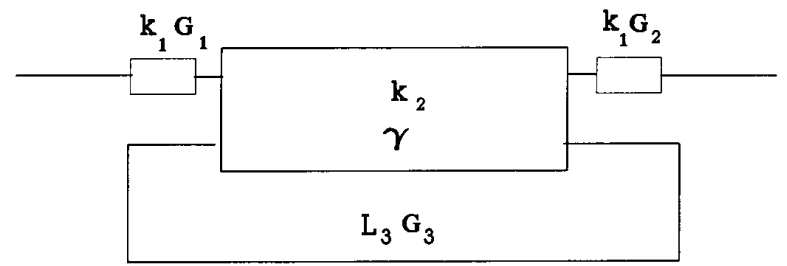

(b)

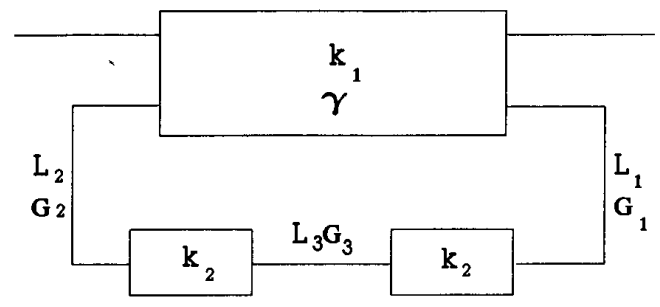

(c)

Fig. 12. Novel descomposition of the double structure in three different rings: (a) The ring is constituted by $l_{1}$ and $l_{2}$. (b) The ring corresponds to $l_{3}$.(c) The ring includes $l_{1}, l_{2}$ and $l_{3}$.

So, all the possible maxima belong to the set of values:

$f=N_{1}\left[\frac{1}{\tau_{1}+\tau_{2}}\right] \cup N_{2}\left[\frac{1}{\tau_{3}}\right] \cup N_{3}\left[\frac{1}{\tau_{1}+\tau_{2}+\tau_{3}}\right]$, where $N_{1}, N_{2}, N_{3} \in \mathbb{N}$

A specific example is going to be tested with experimental results. Let's select the value of $l_{1}=4 m, l_{2}=2 m, l_{3}=40 m$ (the possible maxima are shown in Table I). It would be important to remark that these are frequencies where maxima can be located, but choosing $\left(k_{1}, k_{2}, G_{1}, G_{2}, G_{3}\right)$ the relative amplitude of some of them can be low enough to avoid their presence. The measurements are also shown in Table I and correspond to $k_{1}=0.9 ., k_{2}=0.4, G_{3}=1.44, \alpha_{1}=\alpha_{2}=$ $\alpha_{3}=0.0003 d B / m, \gamma_{1}=\gamma_{2}=0.05$ (Fig. 14(b)).

$k_{1}, \gamma_{1}$ and $G_{1}$ has been calculated utilizing the same process as in the the single ring. To do so, after the desired spectral response has been obtained, the double ring has been physically decomposed into two single rings and (12) and (13) have been used.

A very important conclusion that can be inferred from (36) is that the location of the maxima are determined by $l_{1}, l_{2}, l_{3}$ and $n$, which is the refractive index of the fiber. Although a maximum is fixed at a certain frequency, its relative amplitude in comparison with the others can be controlled by means of changing some parameters such as $k_{1}, k_{2}, G_{1}, G_{2}, G_{3}$. 
TABLE I

FREQUENCY WHERE MAXIMA CAN BE LOCATED FOR $l_{1}=4 m, l_{2}=2 m, l_{3}=40 m$ (FIRST COLUMN). THE Location OF THE MAXIMA FOR THOSE LENGTHS $\mathrm{AND} k_{1}=0.9 ., k_{2}=0.4, G_{3}=1.44, \alpha_{1}=\alpha_{2}=\alpha_{3}=0.0003 \mathrm{~dB} / \mathrm{m}$, $\gamma_{1}=\gamma_{2}=0.05$

\begin{tabular}{|c|c|}
\hline $\begin{array}{l}\text { Frequency (MHz) where the } \\
\text { maxima can be located (equation } \\
\text { (36)) }\end{array}$ & Measurements Frequency Maxima \\
\hline 0 & 0 \\
\hline \multicolumn{2}{|l|}{4.5} \\
\hline 5.2 & 5.2 \\
\hline \multicolumn{2}{|l|}{9} \\
\hline 10.4 & 10.5 \\
\hline \multicolumn{2}{|l|}{13.5} \\
\hline 15.6 & 15.7 \\
\hline \multicolumn{2}{|l|}{18} \\
\hline 20.8 & 21 \\
\hline \multicolumn{2}{|l|}{22.5} \\
\hline 26 & 26 \\
\hline \multicolumn{2}{|l|}{27} \\
\hline 31.2 & 31.3 \\
\hline \multicolumn{2}{|l|}{31.5} \\
\hline \multicolumn{2}{|l|}{34.7} \\
\hline 36 & 36.2 \\
\hline \multicolumn{2}{|l|}{36.4} \\
\hline \multicolumn{2}{|l|}{40.5} \\
\hline 41.6 & 41.2 \\
\hline \multicolumn{2}{|l|}{45} \\
\hline 46.8 & 46.8 \\
\hline 49.5 & \\
\hline
\end{tabular}

This tunability is shown in Fig. 13. The lengths of the loops have been fixed to a certain value $\left(l_{1}=1.5 \mathrm{~m}, l_{2}=\right.$ $\left.5 m, l_{3}=5 m\right)$, and the remainder parameters have been changed. The same frequencies have been converted from maxima to minima, reshaping the transfer function.

\section{Study of the Stability of the System}

In accordance with the three-rings interpretation and the single-ring stability condition, it can be deduced that three conditions are needed to speculate about stability, but they are not enough to confirm it. Those three conditions are:

$$
\begin{aligned}
& G_{1} G_{2}\left(1-k_{1}\right)\left(1-k_{2}\right)\left[10^{-\alpha\left(l_{1}+l_{2}\right) / 10}\right] \\
& \left(1-\gamma_{1}\right)\left(1-\gamma_{2}\right)<1 \\
& G_{3}\left(1-k_{2}\right)\left[10^{-\alpha l_{3} / 10}\right]\left(1-\gamma_{2}\right)<1 \\
& G_{1} G_{2} G_{3}\left(1-k_{1}\right)\left(k_{2}\right)^{2}\left[10^{-\alpha\left(l_{1}+l_{2}+l_{3}\right) / 10}\right] \\
& \quad\left(1-\gamma_{1}\right)\left(1-\gamma_{2}\right)^{2}<1
\end{aligned}
$$

Thus, analysis in the time domain is essential for studying system stability.

\section{Temporal Analysis}

The impulsive response of the system is given by:

$$
h(t)=h_{1}(t)+h_{2}(t)+h_{3}(t)
$$

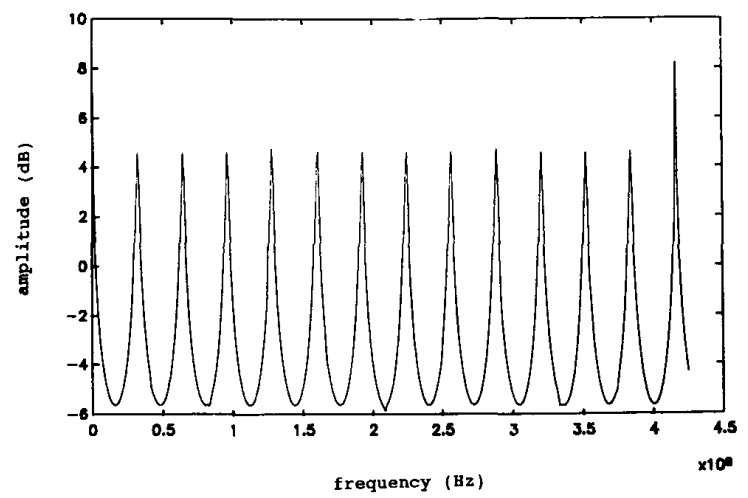

(a)

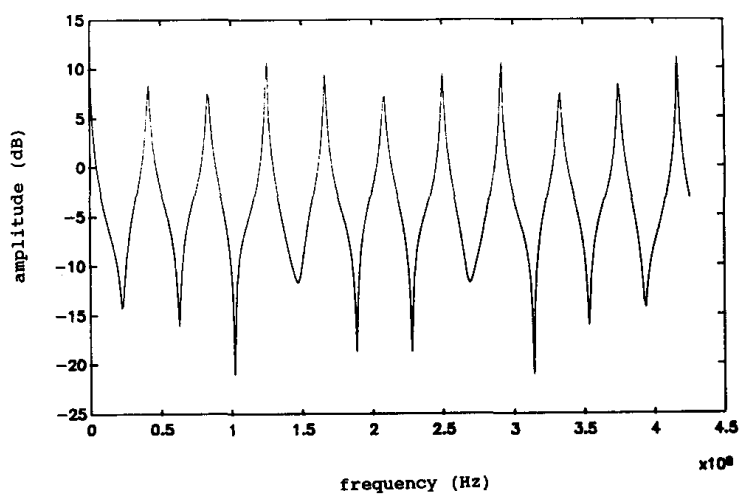

(b)

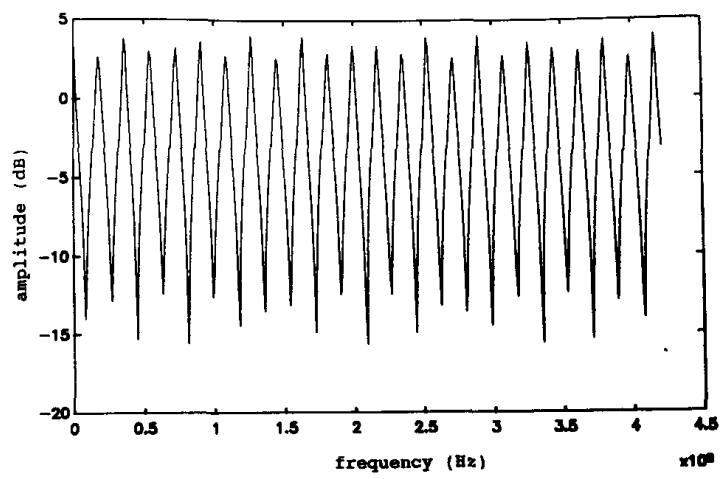

(c)

Fig. 13. Transfer function of the double structure for $l=1.5 \mathrm{~m}, l_{2}=5 \mathrm{~m}$, $l_{3}=5 m, \alpha_{1}=\alpha_{2}=\alpha_{3}=0.0003 \mathrm{~dB} / m, \gamma_{1}=\gamma_{2}=0.5$ and the remainder parameters: (a) $k_{1}=0.5, k_{2}=0.1, G_{1}=1.85, G_{2}=1$. (b) $k_{1}=0.95$, $k_{2}=0.6, G_{1}=1$. $G_{2}=2$. (c) $k_{1}=0.6, k_{2}=0.47, G_{1}=1$., $G_{2}=$ 1.75 .

where $h_{1}(t)=\left(1-k_{1}\right)\left(1-\gamma_{1}\right) \delta(t)$ is the direct term. $h_{2}(t)$ includes all the recirculations along the first ring without entering the ring associated to $l_{3}$ :

$$
\begin{aligned}
h_{2}(t)=\sum_{n=1}^{\infty}\left\{\left[k_{1}\left(1-\gamma_{1}\right)\right]^{2}\left[\left(1-k_{1}\right)\left(1-\gamma_{1}\right)\right]^{n-1}\right. \\
\left.\quad\left[G_{1}^{\prime} G_{2}^{\prime}\left(1-k_{2}\right)\left(1-\gamma_{2}\right)\right]^{n}\right\}\left\{\delta\left(t-n\left(\tau_{1}+\tau_{2}\right)\right)\right\}
\end{aligned}
$$$$
\text { where } G_{i}^{\prime}=G_{i} 10^{-\alpha l_{p} / 10}
$$ 
Finally $h_{3}(t)$ considers the " $n$ " recirculations around the first ring and " $m$ " recirculations to the second ring, crossing " $c$ " times the coupler $k_{2}$.

$$
\begin{aligned}
h_{3}(t)= & \sum_{n=1}^{\infty} \sum_{m=1}^{\infty} \sum_{c=1}^{c=m}\left\{\left[k_{1}\left(1-\gamma_{1}\right)\right]^{2}\left[\left(1-k_{1}\right)\left(1-\gamma_{1}\right)\right]^{n-1}\right. \\
& \left.\times\left[G_{1}^{\prime} G_{2}^{\prime}\left(1-k_{2}\right)\left(1-\gamma_{2}\right)\right]^{n}\right\}\left\{\left[\frac{k_{2}^{2}\left(1-\gamma_{2}\right)}{\left(1-k_{2}\right)}\right]^{c}\right. \\
& \times\left[\left(1-k_{2}\right)\left(1-\gamma_{2}\right)\right]^{m-1}\left[G_{3}^{\prime}\right]^{m} \\
& \left.\times \delta\left[t-n\left(\tau_{1}+\tau_{2}\right)-m \tau_{3}\right]\right\}
\end{aligned}
$$

Although $h(t)$ is constituted by an infinite sum of terms, there is a value of $n=N_{p}$ and $m=M_{p}$ beyond which the terms of the summatory can be neglected. This is only certain when the system is stable. So, (41) would be used to analyze the transient time of this structure once the stability condition is satisfied.

But to assert the stability a further analysis has to be carried out.

The sensitivity of $|H(\Omega)|$ with respect to $\otimes$ (a certain parameter) is:

$$
S_{\otimes}=\frac{\partial|H(\Omega)|}{\partial_{\otimes}}
$$

As $|H(\Omega)|$ is described by $(30), S_{\otimes}$ can be calculated depending on $\otimes$. For instance, if $\otimes=G_{3}$ is chosen:

$$
S_{G_{3}}=\frac{\partial|H(\Omega)|}{\partial G_{3}} \simeq\left[\frac{\partial|H(\Omega)|}{\partial G_{3}}\right]_{\Omega=0} \geq 0
$$

If $G_{3}$ is increased and a negative value of $S_{G_{3}}$ is obtained, $H(\Omega)$ is not describing the system properly because $S_{G_{3}}>0$ for every $G_{3}$, so the system is unstable. This analysis can be done with any other parameter $(\otimes)$, so far as the sign of $S_{\otimes}$ does not change depending on any other parameter (for example: $S_{k 2} \leq 0$ when $G_{1}+G_{2}>G_{3}$, but $S_{k 2} \geq 0$ for $G_{1}+G_{2}<G_{3}$ ).

This method has been used in the numerical program to simulate this structure.

Some measurements have been taken and successfully tested with the theory. Fig. 14(a) and (b) represents the transfer function of the double structure for an EDFA in $l_{2}$, being $k_{1}=0.457, k_{2}=0.4227, l_{1}=2 m, l_{2}=22 m, G_{2}=1.5, \alpha=$ $0.0003 \mathrm{~dB} / \mathrm{m}$ and $\gamma=0.05$.

Fig. 14(c) and (d) shows the transfer function when the EDFA is in $l_{3}$, being $k_{1}=0.9, k_{2}=0.4, l_{1}=4 m, l_{2}=$ $2 m, G_{3}=1.44, \alpha=0.0003 \mathrm{~dB} / \mathrm{m}$ and $\gamma=0.05$.

Fig. 14(a)-(d) shows the good agreement between experimental results and the calculations carried out with the method mentioned above.

\section{FUTURE APPLICATIONS}

Some applications of optical delay lines for signal processing have been introduced in Section $I$, and there is a growing interest in this kind of structures among scientists involved in the field of microwave research [15]. The introduction of optical amplification into delay lines gives some additional

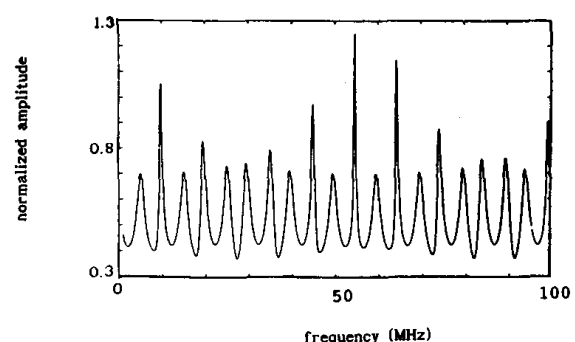

(a)

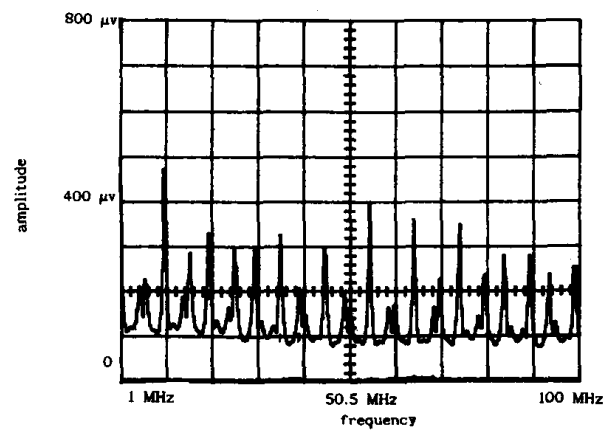

(b)

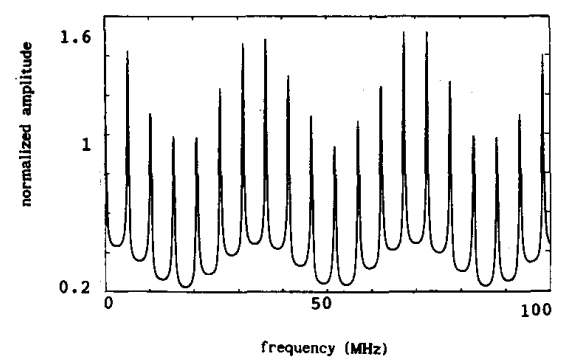

(c)

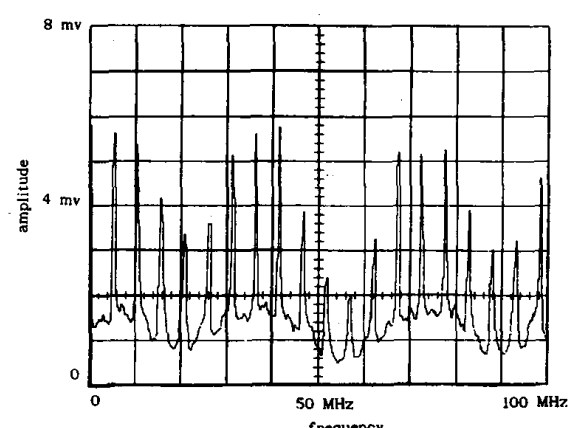

(d)

Fig. 14. Transfer function of the double structure for: EDFA in $1_{2}, 1_{2}=40 m, k_{1}=0.457, k_{2}=0.4227,1_{1}=2 m, 1_{3}=23 m, G_{2}=1.5$, $a_{1}=a_{2}=a_{3}=0.0003 \mathrm{~dB} / \mathrm{m}, \gamma_{1}=\gamma_{2}=0.05$. (a) Calculations. (b) Experimental results. EDFA in $l_{3}, l_{3}=40 \mathrm{~m}, k_{1}=0.9, k_{2}=0.4, l_{1}=4 m, l_{2}=2 m, G_{3}=1.44, \alpha_{1}=\alpha_{2}$ $=\alpha_{3}=0.0003 \mathrm{~dB} / \mathrm{m}, \gamma_{1}=\gamma_{2}=0.05$. (c) Calculations. (d) Experimental results.

features that may be utilized in filtering and simultaneous amplification of multiple, equally spaced transmission bands 
(as needed in CATV systems, where, in some occasions, signals with a bandwidth of $10 \mathrm{MHz}$ are spaced $40 \mathrm{MHz}$ [16]). Other applications may be pulse generation, as shown in Section $\Pi(C)$, or improved transfer function structures for sensing.

It has been recently reported the implementation of a fibre-optic delay-line memory [17]. One of the factors that limits the capacity of this system is the fiber loss in each recirculation. So, the utilization of amplified structures, such as those analyzed in this paper, will avoid this limitation.

In optical frequency division-multiplexing (OFDM), a wide, free-spectral range (FSR) is desirable. Although a single ring has a limited FSR, with the double parallel structures, higher FSR can be achieved by selecting the right relation between their lengths. This approach is similar to the one developed in [18], but with a simpler structure. It would also be also possible to apply our results to integrated optics, obtaining FSR of some GHz.

\section{CONCLUSIONS}

Double and single amplified recirculating optical delay lines have been described theoretically and experimental results have been reported. These configurations have successfully shown their flexibility of design to obtain different transfer functions, depending on the election of certain parameters: gain, length of the loops, and coupling ratios of the couplers.

High peak to peak amplitudes have been achieved. Different cases, to reflect the many possibilities, have been analyzed. A temporal analysis to set the stability conditions and the behavior of the configuration to different input signal has been considered. The saturation region of the amplifier has been delimited, so it has been possible to avoid working within it. It has been concluded that a compromise between gain and speed of response of the system has to be established.

A novel method to understand the behavior of double structures has been developed and succesfully tested with experimental results.

\section{ACKNOWLEDGMENT}

The authors thank Dr. J. Capmany for his encouragement and helpful discussions. They also thank AT\&T.

\section{REFERENCES}

[1] S. A. Newton et al., " Optical fiber V-groove transversal filter," Appl. Phys. Lett. vol. 43, no. 2, pp. 149-151, July 1983.

[2] K. P. Jackson et al., "1-Gbit/s Code generator and matched filter using an optical fiber tapped delay line," Appl. Phys. Lett., vol. 42, no. 7, pp. 556-558, April 1983

[3] M. Our et al., "An optical all-fiber bit-rate limiter" in Proc. ECOC, 1987, pp. 385-388

[4] J. E. Bowers et al., "Filter response of single-mode fibre-recirculating delay lines" Electron. Lett., vol. 18, no. 3, pp.110-111, Feb. 1982.

[5] K. P. Jackson et al., " Optical fiber delay-line signal processing," IEEE Transactions on Microwave Theory and Tech., vol. 33, no. 3, pp. 193-210, March 1985.

[6] S. A. Newton et al., "Single-mode fiber recirculating delay line," SPIE Fiber-optic Tech., vol. 326, pp. 108-115, 1982

[7] R. I. McDonald and R. Nychka, "Differential measurement technique for optical fibre sensors," Electron. Lett., vol. 27, no. 23, pp. 2194-2195, Nov. 1991
[8] A. B. Buckman, "General sensitivity enhancement and common-mode compensation principle for interferometric fiber-optic sensors," J. Ligthwave Technol., vol. 8, no. 10, pp. 1456-1460, Oct. 1990.

[9] D-T. Jong and K. Hotate, "Frequency division multiplexing of optical fiber sensors using an optical delay loop with a frequency shifter," Appl. Opt., vol. 28, no. 7, pp. 1289-1297, April 1989.

[10] M. V. Andres and K. W. H. Foulds, "Optical-fiber resonant rings based on polarization-dependent couplers," J. of Ligthwave Technol., vol. 8, no. 8, pp. 1212-1220, Aug. 1990.

[11] J. Capmany and M. A. Muriel, "A new transfer matrix formalism for the analysis of fiber ring resonators: Compound coupled structures for FDMA demultiplexing," J. of Ligthwave Technol., vol. 8, no. 12, pp. 1904-1919, Dec. 1990

[12] B. Mosheli, "Fibre-optic filters employing optical amplifiers to provide design flexibility," Electron Lett., vol. 28, no. 3, pp. 226-227, Jan. 1992

[13] M. C Vázquez, B. Vizoso, M. López-Amo, and M. A. Muriel "Single and double recirculating delay lines as fibre-optic filters," Electron. Lett. vol. 28, no. 11, pp. 1017-1019, May 1992.

[14] K. Nakagawa, S. Nishi, K. Aida, and E. Yoneda, "Trunk and distribution network application of Erbium-doped fibre amplifiers," J. Lightwave Technol., vol. 9, no. 2, pp. 198-208, Feb. 1991.

[15] G. Grimes, "Microwave fiber-optic delay lines: coming of age in 1992," Microwave Journal, vol. 35, no. 8, pp. 61-69, Aug. 1992.

[16] R. K. Yates et al., Fiber Optics and CATV Business Strategy. Boston: Artech House, 1990, pp. 93.

[17] T. J. Soukup, R. J. Feuerstein, and V. P. Heuring, "Implementation of a fiber-optic delay-line memory," Appl. Opt., vol. 31, no. 17, pp. 3233-3240, June 1992.

[18] Kazuhiro Oda, Norio Takato, and Hiromu Toba, "A Wide-FSR Wave guide Double-Ring Resonator for Optical FDM Transmission Systems," J. Lightwave Technol., vol. 9, no. 6, pp. 728-736, June 1991.

Beatriz Vizoso was born in El Ferrol, Spain in 1967. She received the M.S. degree in Applied Physics from the Universidad Complutense of Madrid in 1990. She is currently a Ph.D. student at the Departamento de Tecnologia Fotónica of the Politechnic University of Madrid (UPM). Her fields of interest are fiber-optic devices and structures.

Carmen Vázquez was born in Madrid, Spain, in 1968. She received the M.S degree in Applied Physics from the Universidad Complutense of Madrid in 1991. She is currently a Ph.D. student at the Departamento de Tecnologia Fotónica of the Politechnic University of Madrid (UPM). Her fields of interest are optical amplifiers and integrated optics.

Rafael Civera was born in Alcublas, Valencia, Spain, 1965. He received the Ingeniero Técnico de Telecomunicación degree in 1987, and the Ingenierio de Telecomunicación degree in 1992, both from the Politechnic University of Madrid (UPM). He is currently a Ph.D. student at the Departamento de Tecnologia Fotónica of the Politechnic University of Madrid (UPM). 
Manuel López-Amo (M'91) was born in Madrid, Spain, in 1960. He received the Ingeniero de Telecomunicación degree in 1985 and the Doctor Ingeniero degree in 1989, both from the Polytechnic University of Madrid (UPM). He is currently an Associate Professor at the Departamento de Tecnologia Fotónica UPM. His fields of interest are integrated optics and fiber-optic structures and systems. Dr. Lopez-Amo is a member of the IEEE.
Miguel A. Muriel (M'84) was born in Burgos, Spain, in 1955. He received the Ingeniero de Telecomunicación degree in 1977 and the Doctor Ingeniero degree in 1980, both from the Polytechnic University of Madrid (UPM). He is now Professor and Head of the Departamento de Tecnologia Fotónica (UPM). Prof. Muriel is a member of the IEEE and OSA 